\title{
Ultrastructure of the reticular basement membrane in asthmatic adults, children and infants
}

\author{
S. Saglani*\#, C. Molyneux*, H. Gong*, A. Rogers*, K. Malmström ${ }^{\star}$, A. Pelkonen`, \\ M. Mäkelä", E. Adelroth ${ }^{+}$, A. Bush" ${ }^{\#}$ D.N.R. Payne ${ }^{\#}$ and P.K. Jeffery*
}

ABSTRACT: Reticular basement membrane (RBM) thickening in asthma is considered to be the result of subepithelial fibrosis. Thus, the RBM in asthma should contain an excess of fibrils identified as interstitial collagen and the ratio of fibril to matrix should be increased above normal levels.

Electron micrographs of the RBM were compared with those of interstitial collagen deeper in the bronchial wall using endobronchial biopsy specimens from adult asthmatics (aged 18-41 yrs $(n=10)$ ), children with difficult asthma (aged 6-16 yrs $(n=10))$, wheezy infants with reversible airflow limitation (aged 0.3-2 yrs $(n=10)$ ) and age-matched nonasthmatic controls: 10 adults, nine children and nine symptomatic infants with normal lung function.

Fibrils in the RBM were significantly thinner (median (range) width 39 (30-52) nm versus 59 (4873) $\mathrm{nm}$ ), and fewer fibrils were banded than in the interstitial collagen (ratio of banded to nonbanded fibrils $0.08(0-0.17)$ versus $0.22(0-1.3))$. The ratio of fibrils to matrix in the thickened RBM of asthmatics did not differ from that of their respective controls $(1.34(0.63-2.49)$ versus 1.18 (0.31-2.6)).

The ratio of fibril to matrix in the thickened reticular basement membrane of asthmatics is normal, and, contrary to what is expected in fibrosis, the fibrils do not resemble those of interstitial collagen.

KEYWORDS: Asthma, reticular basement membrane, subepithelial fibrosis, transmission electron microscopy, ultrastructure

$\mathbf{M}$ orphologically, the subepithelial basement membrane in human airways is composed of a basal lamina (sometimes referred to as the true basement membrane), to which the epithelium is attached. Extending externally from the basal lamina is a lamina reticularis (henceforth referred to as the reticular basement membrane (RBM)). Immunohistochemistry demonstrates that type IV collagen is a major component of the basal lamina [1]. However, without immunostaining, the basal lamina can be visualised only by electron microscopy, since its width $(\sim 80 \mathrm{~nm})$ is below the resolution of the light microscope. By contrast, the $\mathrm{RBM}$ is considerably thicker $(\sim 4 \mu \mathrm{m}$ in healthy adults) and can be seen using light microscopy. The RBM is characteristic of normal humans and other primates, such as monkeys [2]. Histological sections stained with haematoxylin and eosin demonstrate that the RBM is present in all normal healthy individuals, but also that it is homogenously thickened and hyaline in appearance in asthma $[3,4]$.

The abnormal thickening of the RBM begins early in asthma, as early as 4 yrs of age, and is already maximally thickened in children aged 6-16 yrs with severe asthma [5-7].

As immunohistochemistry demonstrates the presence of epitopes for collagen subtypes I, III and $\mathrm{V}$ in the RBM, the abnormal thickening of this layer in asthma has been thought to result from a fibrotic process sometimes described as subepithelial fibrosis [8]. However, these epitopes are part of a morphologically heterogeneous family of glycoproteins that includes both amorphous and fibrillar forms, collectively termed collagen. Fibrosis, as seen in fibrotic lung diseases, is defined on the basis of the accumulation of abnormal amounts of interstitial collagen, or scar tissue. Although the process of interstitial pulmonary fibrosis (IPF), in the lung

\section{AFFILIATIONS}

*Lung Pathology, Depts of Gene Therapy, and

\#Respiratory Paediatrics, Imperial College at the Royal Brompton Hospital, London, UK.

"Dept of Paediatrics, Skin and Allergy Hospital, University of Helsinki Central Hospital, Helsinki, Finland.

+Dept of Respiratory Medicine and Allergy, Umeå University Hospital, Umeå, Sweden.

CORRESPONDENCE

P.K. Jeffery

Lung Pathology

Imperial College

Royal Brompton Hospital

Sydney Street

London

SW3 6NP

UK

Fax: 442073518435

E-mail: p.jeffery@imperial.ac.uk

Received:

May 122005

Accepted after revision:

April 112006

\section{SUPPORT STATEMENT}

This study was supported by Asthma UK (London, UK). 
parenchyma, is considered irreversible, thickening of the epithelial RBM in asthma can be reversed, at least partially, following anti-inflammatory treatment [9] or removal of the asthmatic from an occupational cause [10]. To date, there has been no objective quantitative examination of this key aspect of remodelling in asthma.

Interstitial collagen, and that associated with pulmonary fibrosis, is composed of fibrils of defined width, each with an ultrastructurally characteristic pattern of periodic banding [11]. These criteria were used to test the hypothesis that RBM thickening in asthma, in adults and children, is the result of fibrosis and would, therefore, exhibit an increased ratio of fibril to matrix, in which the fibrils would have the ultrastructural characteristics of interstitial collagen. The aim of the present study was to compare the ultrastructural appearance and width of RBM fibrils to those of interstitial collagen deeper in the bronchial wall. The ultrastructural features and ratio of fibrils to matrix were compared with age-matched controls for adult and paediatric asthmatics and wheezy infants with reversible airflow obstruction. In order to confirm the proposition that interstitial collagen in the bronchial wall and collagen in IPF have a similar ultrastructure, fibril width and banding were also investigated in lung biopsy specimens from five adult cases with cryptogenic fibrosing alveolitis (CFA).

\section{METHODS}

\section{Subjects}

\section{Adults}

Ten nonsmoking adults (median (range) age 27 (18-41) yrs) with mild steroid-naive asthma were compared with 10 healthy nonsmoking nonatopic controls (age 33 (21-42) yrs). Details of these patients have been provided previously [3], and are summarised in table 1. Collagen ultrastructure in open lung biopsy specimens from five adults with histologically diagnosed CFA was also assessed; the clinical details are summarised in table 2 .

\section{Children}

Ten children (age 10 (6-16) yrs) with difficult asthma were compared with nine nonasthmatic paediatric controls (age 11
(7-16) yrs) undergoing bronchoscopy for other respiratory indications, as previously described [5]. Difficult asthma was defined as persistent symptoms requiring rescue bronchodilator therapy on $>3$ days $\cdot$ week $^{-1}$ despite $>1,600 \mu \mathrm{g} \cdot$ day $^{-1}$ inhaled budesonide (or equivalent) and long-acting $\beta_{2}$-agonists and/or regular oral steroids.

Infants

Asthmatic infants were as previously described [12]. Asthma in this group was defined as persistent symptoms of wheeze and/or cough and reduced airways conductance with bronchodilator reversibility. Lung function was measured by whole-body plethysmography. The infant controls were also symptomatic, but exhibited normal lung function. Ten asthmatic infants (age 12 (4-24) months) and nine control infants (age 11.5 (3-24) months) were included.

\section{Bronchoscopy and endobronchial biopsy specimens}

Flexible bronchoscopy was performed in the adults and children, and endobronchial biopsy specimens were taken from the subcarinae of the right lower lobe. Details of the procedures have been described previously for both children [5] and adults [3]. Rigid bronchoscopy was performed in the infants under general anaesthetic as previously described [12]. Informed consent was obtained from the adults and the parents of the infants and children to use the biopsy specimens for research purposes.

\section{Biopsy specimen processing}

Biopsy specimens were fixed in $2.5 \%$ glutaraldehyde in $0.05 \mathrm{M}$ sodium cacodylate buffer ( $\mathrm{pH} 7.4$ ), post-fixed in $1 \%$ osmium tetroxide (in the same buffer), and dehydrated and embedded in epoxy resin (Araldite $\left.{ }^{\circledR}\right)$. Plastic sections (1- $\mu$ m thick) were stained with alkaline toluidine blue. An area of the section that contained epithelium, RBM and subepithelium was selected. Ultra-thin sections $(70 \mathrm{~nm})$ were cut, placed on high-transmission 200-mesh thin-bar copper grids and stained with uranyl acetate and lead citrate. Micrographs were obtained using a Hitachi H7000 (Hitachi, Nissei Sanyo, Tokyo, Japan) transmission electron microscope.

TABLE 1 Clinical details of adults, children and infants with and without asthma

\begin{tabular}{|c|c|c|c|c|c|c|}
\hline & \multicolumn{2}{|c|}{ Adult } & \multicolumn{2}{|c|}{ Child } & \multicolumn{2}{|c|}{ Infant } \\
\hline & Asthma & Control & Asthma & Control & Asthma & Control \\
\hline Subjects n & 10 & 10 & 10 & 10 & 10 & 9 \\
\hline Males $\mathrm{n}$ & 9 & 4 & 6 & 2 & 3 & 2 \\
\hline Age yrs & $28.5(18-41)$ & $38.0(21-45)$ & $12.5(7-16)$ & $10.5(7-16)$ & $0.77(0.4-1.4)$ & $0.96(0.3-2.0)$ \\
\hline $\mathrm{sGaw} \mathrm{s}^{-1} \cdot \mathrm{kPa}^{-1}$ & ND & ND & ND & ND & $1.4(0.6-2.3)$ & $2.7(2.0-3.2)$ \\
\hline Atopy $\mathbf{n}$ & $10 / 10$ & $0 / 10$ & $10 / 10$ & $2 / 10$ & $4 / 10$ & $2 / 9$ \\
\hline ICS dose $e^{\#} \mu \mathrm{g}$ & NA & NA & 800 (400-2000) & NA & NA & NA \\
\hline
\end{tabular}

Data are presented as absolute numbers or median (range). FEV1: forced expiratory volume in one second; \% pred: percentage of the predicted value; PC20: provocative concentration of methacholine causing a $20 \%$ fall in FEV1; sGaw: specific airway conductance; ICS: inhaled corticosteroids; ND: not determined; NA: not available.

\#: budesonide. 


\section{TABLE 2 Clinical details of patients with cryptogenic fibrosing alveolitis}

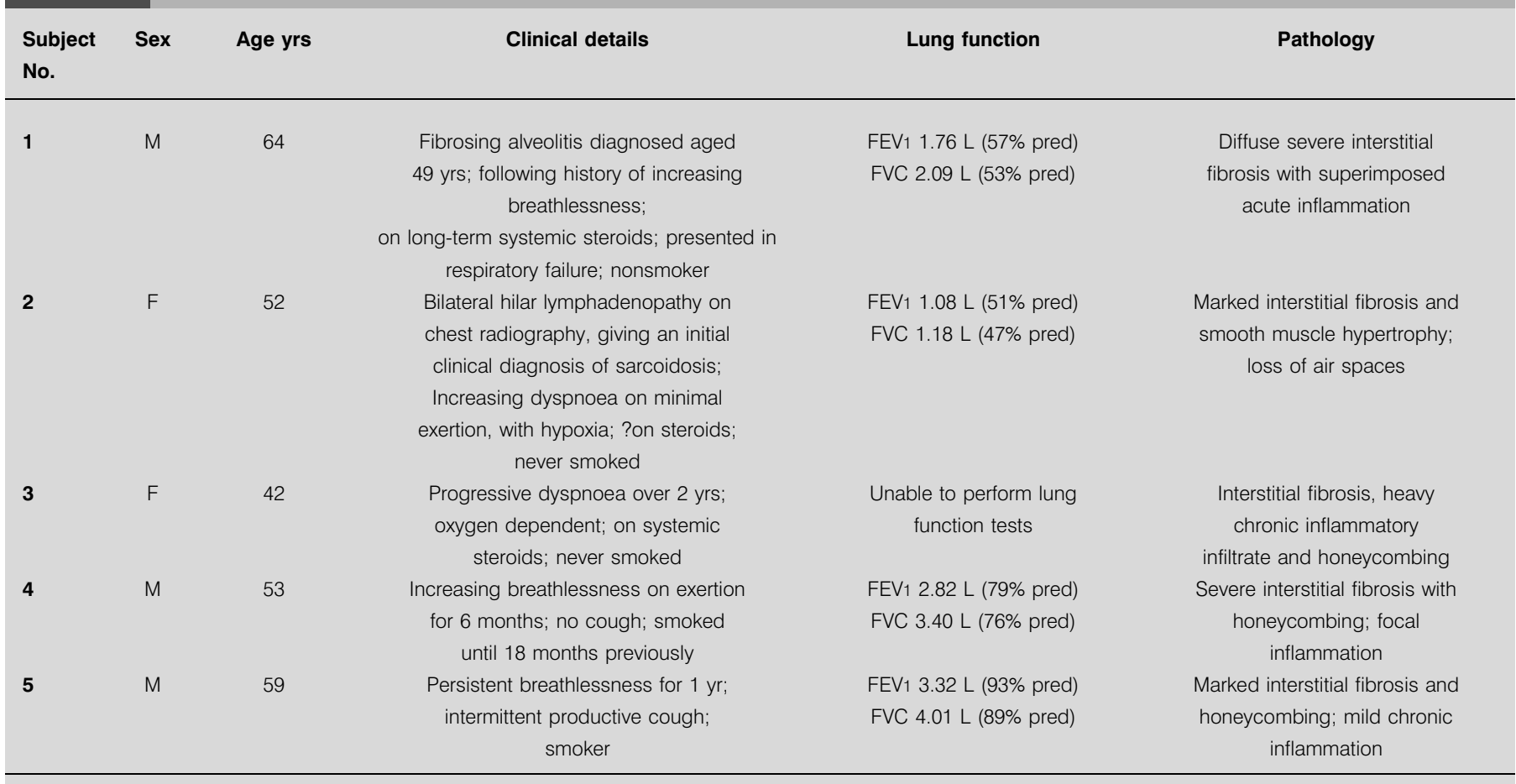

M: male; F: female; FEV1: forced expiratory volume in one second; \% pred: percentage of the predicted value; FVC: forced vital capacity.

\section{Quantification}

From each ultra-thin section, two micrographs were taken of areas of RBM immediately below the basal lamina and two were taken at random from subepithelial areas deep below the RBM and at least $100 \mu \mathrm{m}$ deeper than the basal lamina, the latter representing areas of interstitial collagen. Each micrograph was taken at a magnification of $\times 15,000$. Micrographs were coded and assessed by a single observer who was blind to patient details and the area of the biopsy that had been photographed. The technique of point-counting morphometry was used to assess fibril banding and the ratio of fibril to matrix. A sampling grid of points, arranged $1 \mathrm{~mm}$ apart in a triangular array, was used [13]. The hexagonal area associated with each point represented $\sim 0.75 \mu \mathrm{m}^{2}$. Four randomly selected fields with $\geqslant 200$ points per field were counted on each micrograph [14]. Assessment was made of whether each point was positioned on a fibril or matrix. Furthermore, if the point was on a fibril, an assessment of whether the fibril appeared banded or not at any position along its length was made (fig. 1). Points positioned on the border of fibril and matrix were counted separately, and were divided equally between fibril and matrix counts in the final analysis. In order to assess collagen fibril diameter, four fields each of an area representative of RBM and interstitial collagen were photographed for each patient (magnification $\times 45,000$ ). All micrographs were developed in a similar manner in order to ensure that the contrast remained similar. Five collagen fibrils per field were selected randomly and the width of each fibril was measured using Image Pro 4 software with a threshold autotracer (Media Cybernetics, Inc., Silver Spring, MD, USA).
Twenty fibrils were measured in areas representing RBM and interstitial collagen, and the results expressed as a mean.

In the CFA patients, collagenous areas of lung parenchyma with preserved alveolar architecture were selected and electron micrographs and measurements were made using the methods described previously.

\section{Statistical analyses}

The ratio of banded to nonbanded fibrils was calculated for the RBM and interstitial collagen, and the ratio of fibrils to matrix was calculated for the RBM in each age group. Nonparametric tests were applied to test for inter-group differences. Comparison between all groups was made using the Kruskal-Wallis test, followed by a Mann-Whitney U-test if a significant difference $(p<0.05)$ was found. The Bonferroni correction was introduced for multiple comparisons. Variability of counts was calculated using the percentage coefficient of variation.

\section{Variability}

The intra-observer error of repeat measurement of performing three separate counts of fibril banding on the same micrograph on different occasions was $<5 \%$. Intra-section variability, assessed by taking up to six micrographs from a region and calculating the cumulative mean of counts of the micrographs, revealed that two micrographs provided data representative of fibril banding. The percentage coefficient of variation for the ratio of fibril to matrix measured on the same micrograph on separate occasions was $2.4 \%$ for RBM and $2.7 \%$ for interstitial collagen. 

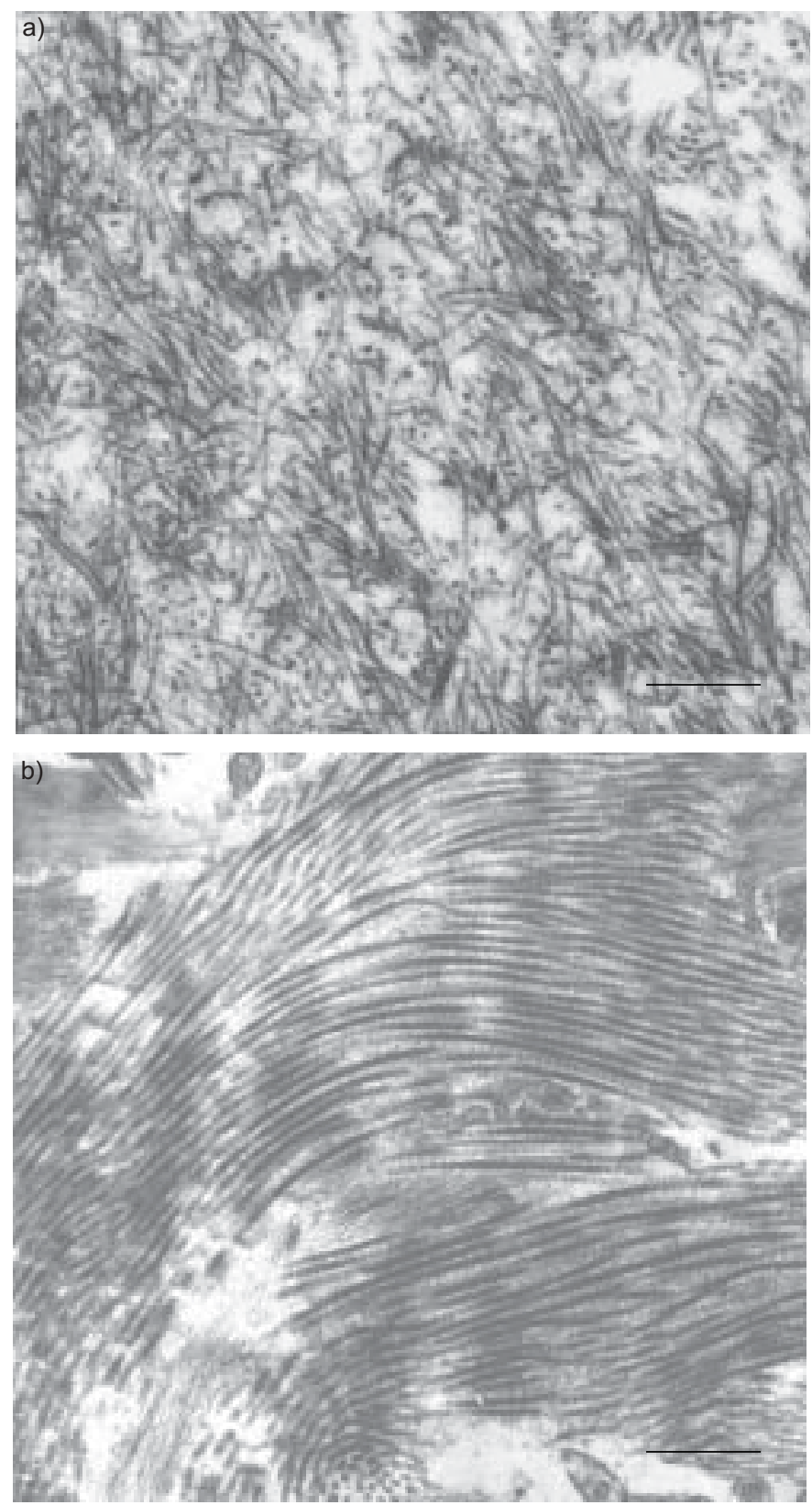

FIGURE 1. Electron micrographs showing the ultrastructural appearance of the bronchial: a) reticular basement membrane (RBM); and b) deeper interstitial collagen. The collagen fibrils are more obviously banded and thicker than the RBM fibrils. Scale bar $=500 \mathrm{~nm}$

\section{RESULTS}

Two features of fibril ultrastructure were evaluated, fibril width and banding (fig. 1). These features in RBM and interstitial collagen were compared in all age groups.

\section{Fibril width in the RBM and interstitial collagen}

Fibril widths were significantly lower in the RBM compared with interstitial collagen in all age groups in both asthma and controls. Infant median (range) fibril width was $47(37-50) \mathrm{nm}$ in the RBM versus $59(48-69) \mathrm{nm}$ in interstitial collagen $(\mathrm{p}<0.001)$. In children, fibril width was $36(31-42) \mathrm{nm}$ in the
RBM versus $54(48-69) \mathrm{nm}$ in interstitial collagen $(\mathrm{p}<0.001)$. Adult fibril width was 36 (30-49) nm in the RBM versus 61 (4874) $\mathrm{nm}$ in interstitial collagen ( $\mathrm{p}<0.001$; fig. 2 ).

\section{Ratio of banded to nonbanded fibrils}

There were significantly fewer banded fibrils in the RBM compared with interstitial collagen in all age groups in both asthma and controls (fig. 3).

\section{Ratio of fibrils to matrix in the RBM}

The ratio of fibrils to matrix in the RBM of the adult, paediatric and infant asthmatics was similar to that of the age-matched controls. The median (range) ratio in adult asthmatics versus controls was $0.94(0.60-1.46)$ versus $0.64(0.47-1.10)(p=0.09)$. The ratio in paediatric asthmatics versus controls was 1.24 $(0.63-2.49)$ versus $1.31(0.50-2.57)(\mathrm{p}=0.96)$ and the ratio in infant asthmatics versus controls was $1.67(0.68-3.08)$ versus 1.29 (0.31-2.96) ( $\mathrm{p}=0.74$; fig. 4).

\section{Ratio of fibrils to matrix in bronchial interstitial collagen}

In order to assess whether a pattern of a more classical interstitial fibrosis was present within the submucosa of asthmatic tissue, the ratio of fibrils to matrix in the deeper interstital collagen was compared in the adult asthmatics and controls. There was no difference in the ratio of fibrils to matrix in the deeper interstitial collagen between adult asthmatics and controls (1.21 (0.71-1.78) versus $1.18(0.81-2.15)(\mathrm{p}=\mathrm{NS}))$.

\section{Fibril width and banding in bronchial interstitial collagen and parenchymal collagen in CFA}

At all ages, interstitial collagen fibril width was similar to that of the parenchymal collagen of CFA patients. The median (range) collagen width in infants was 59 (48-69) nm versus 54 $(48-69) \mathrm{nm}$ in children, 61 (48-74) $\mathrm{nm}$ in adults and 55 (4363) $\mathrm{nm}$ in CFA ( $p=N S$; fig. 5). All collagen fibrils in CFA patients were banded.

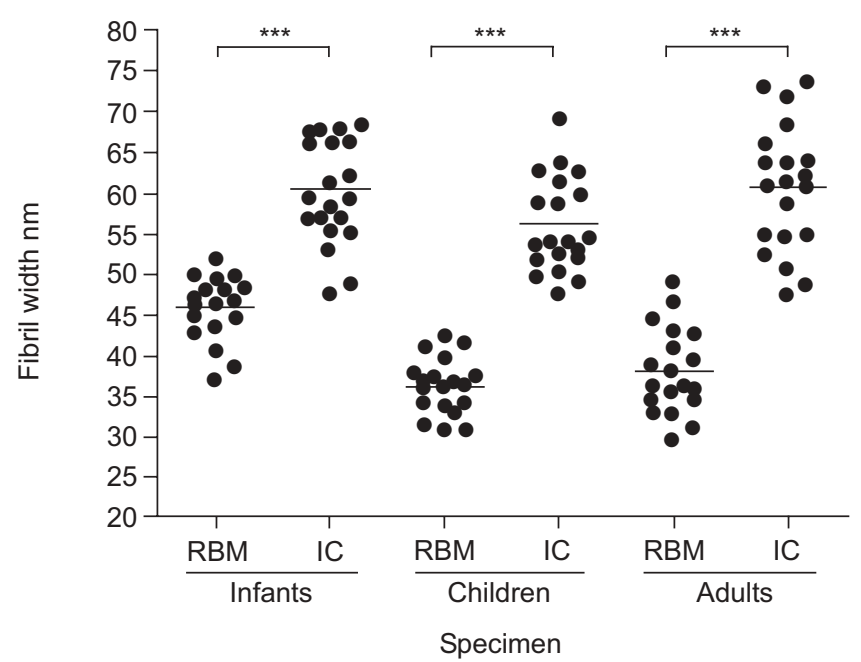

FIGURE 2. Fibril width in the reticular basement membrane (RBM) compared with interstitial collagen (IC) in infants, children and adults. Horizontal bars represent medians. ${ }^{* *}: \mathrm{p}<0.001$. 


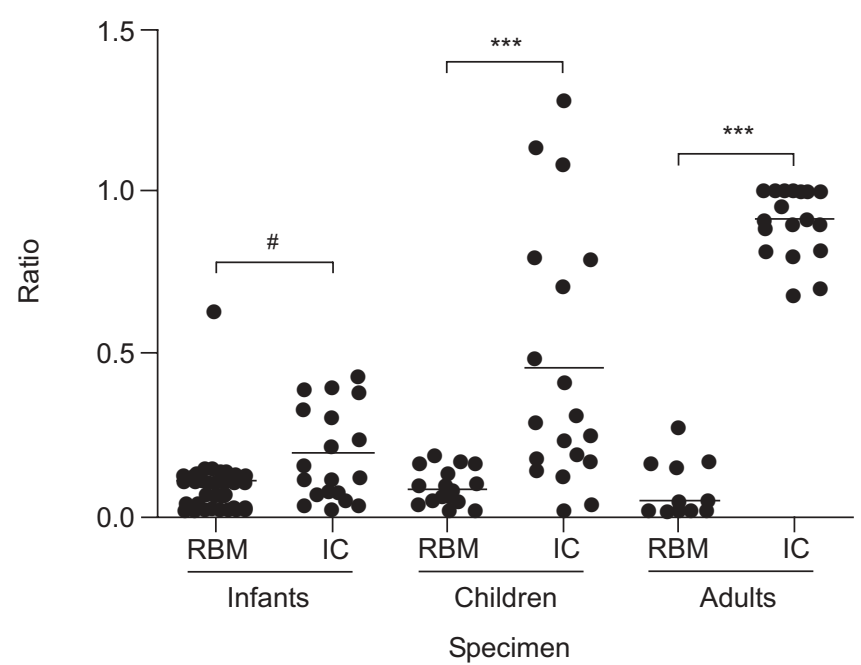

FIGURE 3. Ratio of banded to nonbanded fibrils in the reticular basement membrane (RBM) and interstitial collagen (IC) in infants, children and adults. There are more banded fibrils as the ratio increases. Horizontal bars represent medians. \#: $p<0.03$; ${ }^{* *}: p<0.001$.

\section{Fibril width in RBM in asthma and parenchymal collagen in CFA}

The RBM fibril width, in both adult and paediatric asthmatics, was significantly lower than the parenchymal collagen fibril width of CFA patients (36 (30-43) nm in adult asthma versus 37 (31-41) nm in child asthma and 55 (43-63) nm in CFA $(\mathrm{p}<0.01$ for adult and child asthma versus CFA)). The RBM fibril width was also lower in infants than the parenchymal collagen fibril width of CFA patients, but this difference was not significant (48 (43-52) nm in infants versus $55(43-63) \mathrm{nm}$ in CFA $(\mathrm{p}=\mathrm{Ns})$ ) (fig. 6).

\section{DISCUSSION}

The present study has shown that the ultrastructure of the $\mathrm{RBM}$ is distinct to that of bronchial interstitial collagen, the

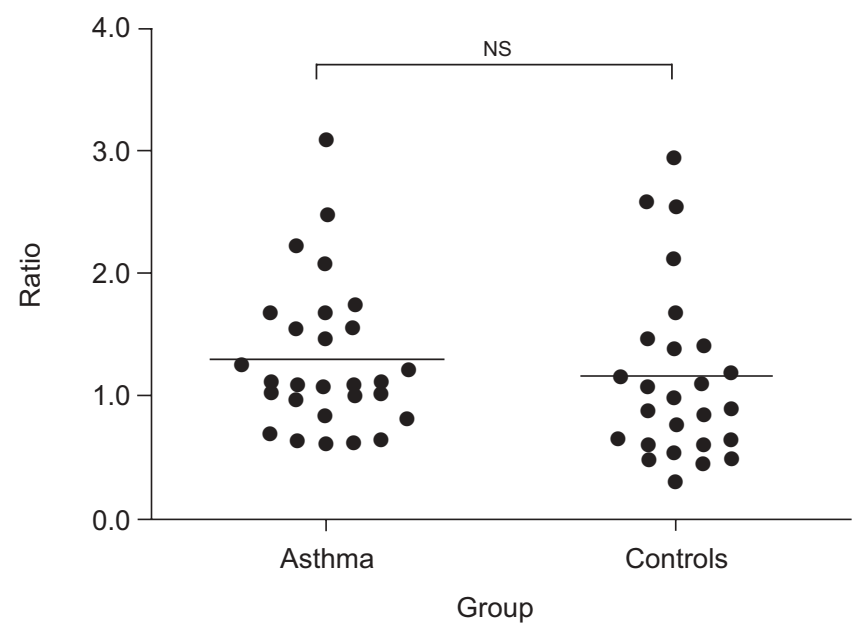

FIGURE 4. Ratio of fibrils to matrix in the reticular basement membrane in asthmatics compared with age-matched controls (all ages together). There are more fibrils as the ratio increases. Horizontal bars represent medians.

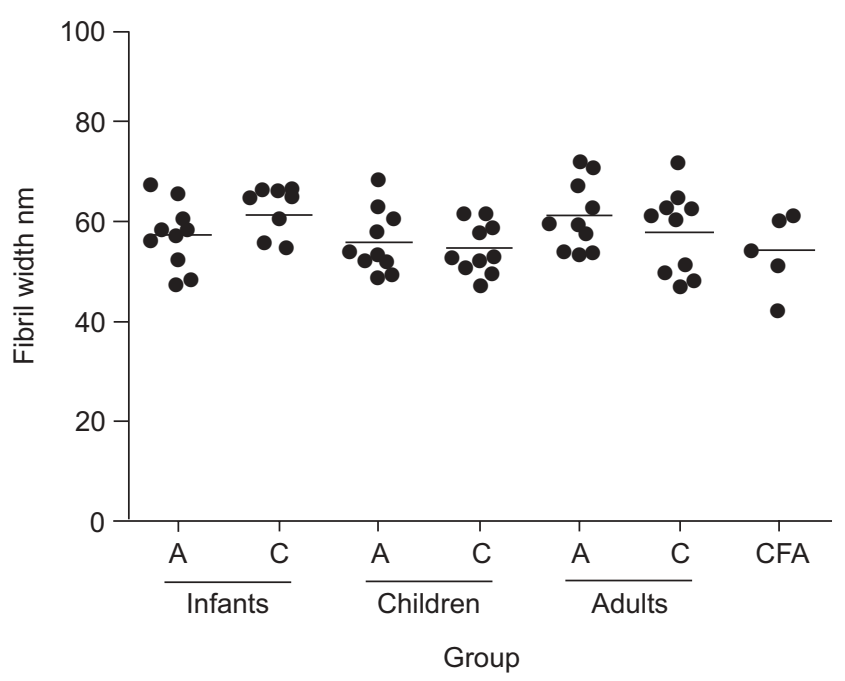

FIGURE 5. Interstitial collagen fibril width in infants, children and adults compared with adults with cryptogenic fibrosing alveolitis (CFA). Horizontal bars represent medians. A: asthma; C: control.

latter being similar to the alveolar wall collagen of pulmonary fibrosis. Even when the RBM is thickened, as in asthma, the fibrils are thinner and less obviously banded than those of airway wall interstitial collagen. Moreover, the ratio of fibrils to matrix in asthma, at all ages, remains unchanged compared with nonasthmatic controls. The present ultrastructural data do not support the hypothesis that thickening of the RBM in asthma results from the same fibrotic process as that seen in fibrotic lung disease.

Collagen is a universal term applied to a family of glycoproteins and includes distinct structural forms such as interstitial collagen, reticulin and constituents of basal laminae [1]. In mammals, the fibrils of interstitial collagen are thicker, with a diameter ranging 10-500 $\mathrm{nm}$ (mean $60 \mathrm{~nm}$ ), than those of reticulin and usually follow a wavy course without branching [11]. Additionally, interstitial collagen contains fibrils that each have characteristic transverse bands termed D-periodicity with a repetitive band frequency of $\sim 64-70 \mathrm{~nm}$ [15]. In contrast, reticulin is composed of fibrils that form a relatively tangled loosely packed network of fine fibrils with a diameter of $\sim 20$ $40 \mathrm{~nm}$ and absent or at least much less obvious D-periodicity [16]. The term subepithelial fibrosis has been suggested and is variously applied by different investigators to describe the characteristic thickening of the RBM layer in asthma [8]. To many, the term implies that it is the addition of interstitial collagen that contributes to the RBM thickening, suggesting a fibrotic process similar to that described in IPF.

A previous quantitative study assessed the RBM electron microscopically, but only to determine its thickness in asthma compared with controls [17]; none have assessed its ultrastructural composition. The present authors acknowledge that the descriptor reticular of RBM per se already implies a distinction from interstitial collagen. However, it has been demonstrated for the first time, using quantitative and ultrastructural techniques, that the composition of the normal bronchial RBM, as well as of that which is thickened in asthma, is ultrastructurally different from the banded thicker fibrils of 


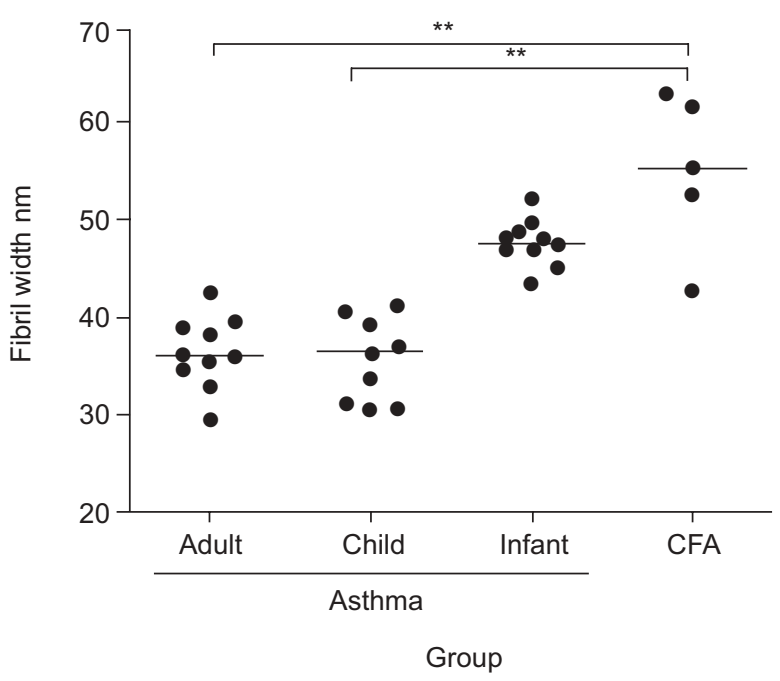

FIGURE 6. Reticular basement membrane fibril width in asthmatics of all ages compared with parenchymal collagen fibril width in adults with cryptogenic fibrosing alveolitis (CFA). Horizontal bars represent medians. **: $p<0.01$

interstitial collagen characteristic of fibrotic scarring and that seen in parenchymal collagen from IPF patients. This ultrastructural distinction, together with the lack of a relative increase in the fibrillary component and the nonprogressive nature of the thickening, which has previously been shown to be already maximal in asthmatic children [5], indicates that the process of RBM thickening in asthma is different to the fibrosis characteristic of IPF.

Experimental studies provide support for the present interpretation. In allergen-challenged mice, silver stains (designed to highlight reticulin) demonstrate that subepithelial reticulin in the airways increases three-fold compared with that of sham-challenged mice and that it is this component that contributes to the increased RBM thickness in this animal model of asthma [18]. Moreover, it has additionally been shown that both fibrils and matrix increase in equal proportion in human asthma such that the thickened RBM in asthma, in the child or adult, has the same ultrastructural appearance as that of age-matched controls. It has previously been shown that the RBM is present and thickened in both adults [3] and children with asthma $[5,6]$, and it has recently been reported that the RBM is developed, but not yet thickened, in infants aged $<24$ months [12]. In the present article, it has been shown that age does not influence the ultrastructural composition of the RBM, in either asthmatics or nonasthmatic controls. Also, the ultrastructural appearance of the RBM might differ in patients with more severe disease, defined by the presence of persistent airflow limitation [19]. In the present study, this was addressed in children, who all exhibited difficult asthma. Although numbers were small (six with and four without persistent airflow limitation), there were no differences in RBM ultrastructure between the subjects with and without persistent airflow limitation.

One potential criticism of the present study is that the RBM was assessed using only transmission electron microscopy and without the application of immunohistochemical and silver staining techniques to identify reticulin. To apply these, in repetition of previous studies [20], would have required additional biopsy specimens, processed using different techniques to those used for electron microscopy. Sufficiently large numbers of biopsy specimens, particularly from children, were neither available nor ethically approved. Neither was it considered that this was required to reach the present conclusions, since electron microscopy so clearly distinguishes reticulin from fibrils of interstitial collagen without need for special stains [21]. Many studies have used immunohistochemical staining techniques to assess which collagen subtypes are increased in the RBM in asthma [17]. These researchers have shown that the main components of the RBM are collagen subtypes III [8, 22] and V [8, 17], which is in keeping with increased reticulin, since, immunohistochemically, reticulin fibrils express the epitopes for collagen types III [21, 23] and V [24], whereas interstitial collagen fibrils most closely resemble collagen type I $[1,23]$. A recent study has reported increased collagen subtype III in both adult and paediatric asthmatics [25]. This is probably not due to an increased density of type III collagen, but rather the result of an increase in the total thickness of the RBM. Moreover, such a light microscopic study, in which immunostaining is performed, does not permit assessment of the ratio of fibrils to matrix in the thickened asthmatic RBM; for this, electron microscopy is required. As undertaken previously, but using snap-frozen material [8], immunostaining of paraffin wax-embedded biopsy specimens was performed for collagen subtypes I, III and V in cases in which tissue was available; this was in addition to the ultrastructural assessment of all plastic-embedded biopsy specimens. The clear discrimination that was observed between RBM reticulin and deeper interstitial collagen by electron microscopy was not reflected by similarly distinct distributions of collagen type III. Also, the variability associated with staining for collagen types I and V in the biopsy specimens that had been processed into paraffin wax made their interpretation unconvincing.

Thus, although it is acknowledged that the epithelial RBM of asthma stains for the epitopes that mark collagen, the present authors consider that electron microscopy is a technique which offers an additional and novel means of examining and comparing the structural components of the RBM and interstitial collagen in asthma, and that there are marked differences in structure between the composition of the RBM and deeper bronchial or parenchymal interstitial collagen, the latter taken from patients with fibrosing lung disease.

Finally, myelofibrosis is a condition involving increased bone marrow deposition of collagen and reticulin [26]. In bone marrow disease, the term reticulin fibrosis or collagen fibrosis is used [27], and a variable response of reticulin fibrosis to corticosteroids has been reported [27]. Even in patients with CFA, variable amounts of both type I and III collagen are present, and patients with a larger proportion of type III collagen show a better response to steroid therapy [28]. This is consistent with evidence that long-term steroid therapy can result in a reduction in RBM thickness in asthma [29]. The present authors suggest that a distinction between reticulin fibrosis and collagen fibrosis be recognised in asthma, and that that the former term would be more appropriate and specific than the term subepithelial fibrosis. Alternatively, the term 
reticular basement membrane thickening, although less specific, is accurate, less likely to confuse and implies an as yet equivocal mechanism for explaining its thickening in asthma.

In summary, it has been shown, by ultrastructural examination, that reticular basement membrane thickening in asthma represents not interstitial collagen but rather increased deposition of reticulin and matrix in equal and normal proportion. These findings, in children and adults, led to the conclusion that the reticular basement membrane thickening in asthma represents an increased quantity of lamina reticularis of the same composition as that found in healthy individuals. Although immunostaining identifies epitopes usually associated with collagen, the present ultrastructural data demonstrate distinctions from the interstitial collagen associated with fibrosis and challenge the notion that the reticular basement membrane and its thickening in asthma are the result of a fibrotic process of the kind seen in fibrotic lung disease.

\section{ACKNOWLEDGEMENTS}

The authors would like to thank A. Dewar and the Dept of Histopathology (Royal Brompton Hospital, London, UK) for their help in identifying the subjects with cryptogenic fibrosing alveolitis.

\section{REFERENCES}

1 Montes GS. Structural biology of the fibres of the collagenous and elastic systems. Cell Biol Int 1996; 20: 15-27.

2 Evans MJ, Fanucchi MV, Baker GL, et al. The remodelled tracheal basement membrane zone of infant rhesus monkeys after 6 months of recovery. Clin Exp Allergy 2004; 34: 1131-1136.

3 Jeffery PK, Godfrey RW, Adelroth E, Nelson F, Rogers A, Johansson SA. Effects of treatment on airway inflammation and thickening of basement membrane reticular collagen in asthma. A quantitative light and electron microscopic study. Am Rev Respir Dis 1992; 145: 890-899.

4 Jeffery PK. Remodeling in asthma and chronic obstructive lung disease. Am J Respir Crit Care Med 2001; 164: S28-S38.

5 Payne DN, Rogers AV, Adelroth E, et al. Early thickening of the reticular basement membrane in children with difficult asthma. Am J Respir Crit Care Med 2003; 167: 78-82.

6 Barbato A, Turato G, Baraldo S, et al. Airway inflammation in childhood asthma. Am J Respir Crit Care Med 2003; 168: 798-803.

7 Pohunek P, Warner JO, Turzikova J, Kudrmann J, Roche WR. Markers of eosinophilic inflammation and tissue re-modelling in children before clinically diagnosed bronchial asthma. Pediatr Allergy Immunol 2005; 16: 43-51.

8 Roche WR, Beasley R, Williams JH, Holgate ST. Subepithelial fibrosis in the bronchi of asthmatics. Lancet 1989; 1: 520-524.

9 Ward C, Pais M, Bish R, et al. Airway inflammation, basement membrane thickening and bronchial hyperresponsiveness in asthma. Thorax 2002; 57: 309-316.
10 Saetta M, Di Stefano A, Maestrelli P, et al. Airway mucosal inflammation in occupational asthma induced by toluene diisocyanate. Am Rev Respir Dis 1992; 145: 160-168.

11 Parry DA, Craig AS. Growth and development of collagen fibrils in connective tissue. In: Ruggeri A, Motta PM, eds. Ultrastructure of the Connective Tissue Matrix. Boston, MA, Martinus Nijhoff Publishers, 1984; pp. 34-64.

12 Saglani S, Malmstrom K, Pelkonen AS, et al. Airway remodelling and inflammation in symptomatic infants with reversible airflow obstruction. Am J Respir Crit Care Med 2005; 171: 722-727.

13 Aherne WA, Dunhill MS. Point counting and the estimation of volume fraction. In: Aherne WA, Dunhill MS, eds. Morphometry. 3rd Edn. London, Edward Arnold Publishers, 1987; pp. 33-44.

14 Howard CV, Reed MG. Estimation of component volume and volume fraction. In: Howard CV, Reed $\mathrm{MG}$, eds. Unbiased Stereology. Three-Dimensional Measurement in Microscopy. 1st Edn. Oxford, BIOS Scientific Publishers, 1998; pp. 55-65.

15 Ushiki T. Collagen fibers, reticular fibers and elastic fibers. A comprehensive understanding from a morphological viewpoint. Arch Histol Cytol 2002; 65: 109-126.

16 Ohtani O, Kikuta A, Ohtsuka A, Murakami T. Organisation of the reticular network of rabbit Peyer's patches. Anat Rec 1991; 229: 251-258.

17 Wilson JW, Li X. The measurement of reticular basement membrane and submucosal collagen in the asthmatic airway. Clin Exp Allergy 1997; 27: 363-371.

18 Blyth DI, Wharton TF, Pedrick MS, Savage TJ, Sanjar S. Airway subepithelial fibrosis in a murine model of atopic asthma - suppression by dexamethasone or antiinterleukin-5 antibody. Am J Respir Cell Mol Biol 2000; 23: 241-246.

19 Payne DN, Qiu Y, Zhu J, et al. Airway inflammation in children with difficult asthma: relationships with airflow limitation and persistent symptoms. Thorax 2004; 59: 862-869.

20 Fleischmajer R, Jacobs L 2nd, Perlish JS, Katchen B, Schwartz E, Timpl R. Immunochemical analysis of human kidney reticulin. Am J Pathol 1992; 140: 1225-1235.

21 Montes GS, Krisztan RM, Shigihara KM, Tokoro R, Mourao PA, Junqueira LC. Histochemical and morphological characterization of reticular fibers. Histochemistry 1980; 65: 131-141.

22 Chu HW, Halliday JL, Martin RJ, Leung DY, Szefler SJ, Wenzel SE. Collagen deposition in large airways may not differentiate severe asthma from milder forms of the disease. Am J Respir Crit Care Med 1998; 158: 1936-1944.

23 Magloire H, Joffre A, Grimaud JA, Herbage D, Couble ML, Chavrier C. Distribution of type III collagen in the pulp parenchyma of the human developing tooth. Light and electron microscope immunotyping. Histochemistry 1982; 74: 319-328.

24 Stenman S, Vaheri A. Distribution of a major connective tissue protein, fibronectin, in normal human tissues. J Exp Med 1978; 147: 1054-1064. 
25 Fedorov IA, Wilson SJ, Davies DE, Holgate ST. Epithelial stress and structural remodelling in childhood asthma. Thorax 2005; 60: 389-394.

26 Johnston JB, Dalal BI, Israels SJ, et al. Deposition of transforming growth factor- $\beta$ in the marrow in myelofibrosis, and the intracellular localization and secretion of TGF- $\beta$ by leukemic cells. Am J Clin Pathol 1995; 103: 574-582.

27 Bass RD, Pullarkat V, Feinstein DI, Kaul A, Winberg CD, Brynes RK. Pathology of autoimmune myelofibrosis. A report of three cases and a review of the literature. Am J Clin Pathol 2001; 116: 211-216.

28 Kirk JM, Heard BE, Kerr I, Turner-Warwick M, Laurent GJ. Quantitation of types I and III collagen in biopsy lung samples from patients with cryptogenic fibrosing alveolitis. Coll Relat Res 1984; 4: 169-182.

29 Ward C, Walters H. Airway remodelling: the influence of corticosteroids. Curr Opin Allergy Clin Immunol 2005; 51: $43-48$. 